\title{
Observation of a Stable Monomeric N-Methylene Aromatic Amine†
}

\author{
Giancarlo Verardo, Sabina Cauci, and Angelo G. Giumanini* \\ Department of Chemistry, University of Udine, I-33100 Udine, Italy
}

The first free $\mathrm{N}$-methyleneaniline stable under ordinary conditions was authenticated by spectroscopic and analytical data and some of its reactions were observed; a room temperature equilibrium between another free $\mathrm{N}$-methylene aromatic amine and its oligomeric species is reported.

We have recently defined many of the structural details of the monomer, dimer, ${ }^{1}$ trimer, ${ }^{2}$ and tetramer ${ }^{3}$ of the Schiff base derived from aniline and formaldehyde. However, there is no literature report to date about any free monomeric $N$-methylene aromatic amine stable under ordinary conditions in the solid or liquid state, authenticated by analytical and spectroscopic data. 4

We report the preparation and reactions of $N$-methylene2,6-di-isopropylaniline (1), the first stable characterised monomeric $N$-methylene aromatic amine. It is easily prepared by heating equimolar amounts of polyoxymethylene (2) and 2,6-di-isopropylaniline (3) until the former has completely dissolved at $c a .100{ }^{\circ} \mathrm{C}$. $¥$ Product $(\mathbf{1})$, which correctly analysed for $\mathrm{C}_{13} \mathrm{H}_{19} \mathrm{~N}$, is a colourless liquid, its positive ion electron impact mass spectrum showed a parent ion at $\mathrm{m} / \mathrm{z} 189$, with sequential losses of a methyl group $(\mathrm{m} / \mathrm{z} 174)$ and propene $\S$

$\dagger$ Part of the series: 'Revisitation of the Reaction between Aniline and Formaldehyde.'

¥ Water formed and any excess of (2) can be eliminated by evaporation in vacuo. The colourless reaction product is then distilled under reduced pressure $\left(\mathrm{ca} .50^{\circ} \mathrm{C} / 0.1 \mathrm{Torr}\right.$; quantitative yield). G.c. analysis (capillary column, $25 \mathrm{~m}$ long, $0.1 \mathrm{~mm}$ i.d., $\mathrm{SE} 30 ; \mathrm{H}_{2}$ carrier, injector temp. $200^{\circ} \mathrm{C}$, flame ionisation detector, column temp. from 80 to $200^{\circ} \mathrm{C}, 10^{\circ} \mathrm{C} / \mathrm{min}$ ) combined with electron impact mass spectrometry reveals the presence of some (less than $3 \%$ ) unreacted (3), which appears difficult to remove either by conventional separation methods or further reaction with excess of (2).

$\S$ The formal loss of 57 units from the parent ion of (1) may be either a $\mathrm{C}_{4} \mathrm{H}_{9}$ or a $\mathrm{C}_{3} \mathrm{H}_{7} \mathrm{~N}$ fragment. We prepared $\mathrm{N}$-dideuteriomethylene-2,6di-isopropylaniline, which upon electron impact at $70 \mathrm{eV}$ lost both 59 units $\left(M^{+}-\mathrm{Et}\right.$, then $\left.-\mathrm{CD}_{2} \mathrm{~N}\right)$ and to a larger extent 57 units $\left(\mathrm{M}^{+-}\right.$ $-\mathrm{Me}$, then $\left.-\mathrm{C}_{3} \mathrm{H}_{6}\right)$. These observations indicated deep skeletal rearrangements of the parent ion not involving the $\mathrm{N}=\mathrm{CD}_{2}$ function prior to fragmentation. $(m / z 132$, base peak) at $70 \mathrm{eV}$. The i.r. spectrum exhibited a practically identical pattern in the aromatic out-of-plane bending region of $\mathrm{CH}$ bonds $\left(756 \mathrm{~s}\right.$ and $\left.785 \mathrm{~m} \mathrm{~cm}^{-1}\right)$ as compared with (3) (740s and $\left.782 \mathrm{~cm}^{-1}\right)$ and two of the strongest bands in the finger-print region were almost coincidental in position [ $1042 \mathrm{~m}$ and 1460 s shouldered unresolved multiplet, in (1)]; the bands of (3) at $1620 \mathrm{~m}$ and the $\mathrm{NH}_{2}$ stretching doublet centred at $3440 \mathrm{~m}-\mathrm{w}$ disappeared. A sharp band appeared at $1647 \mathrm{~s} \mathrm{~cm}^{-1}$ for (1). The ${ }^{1} \mathrm{H}$ n.m.r. spectrum of (1) $\left(\mathrm{CDCl}_{3}\right)$ showed resonances of the nonequivalent methylene protons at very low field, $\delta 7.72$ and 7.35 (AB-quartet, $2 J_{\mathrm{H}-\mathrm{H}} 18.3 \mathrm{~Hz}$ ), beyond the aromatic complex multiplet, centred at $\delta 7.10(3 \mathrm{H})$, in addition to the expected aliphatic pattern, $\delta 2.93(\mathrm{CH}, 2 \mathrm{H}$, septet, $J 6.9 \mathrm{~Hz})$, $1.15\left(\mathrm{CH}_{3}, 12 \mathrm{H}\right.$, doublet $)$. I Space filling molecular models clearly evidenced the impossibility of positioning the methylene group atoms in the plane of the ring and the nitrogen atom; the two hydrogen atoms as a result end up in different environments, and rotation about the $\mathrm{C}-\mathrm{N}$ bond is hindered.

The nature of the methylene group is confirmed by ${ }^{13} \mathrm{C}$ n.m.r. spectroscopy: we found that the resonance of the methylene carbon falls some $646 \mathrm{~Hz}$ below the resonance of the highest field aromatic carbon; the corresponding signal for

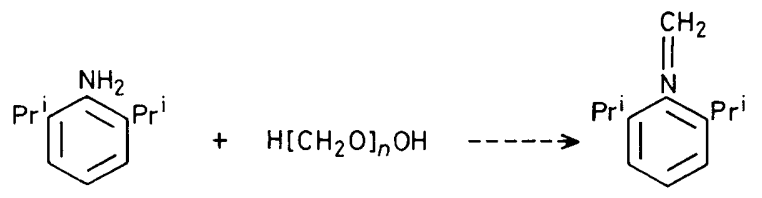

(3)

(2)

(1)

I The ${ }^{2 J}$ value for the methylene protons is very close to the figures reported for an aliphatic $N$-methyleneimine. ${ }^{6}$ 
trimeric $N$-methyleneaniline (1,3,5-triphenylhexahydro-symtriazine) is located some $1006 \mathrm{~Hz}$ above the resonance of the highest field aromatic carbon (respectively 436.9 and $1541 \mathrm{~Hz}$ below the ${ }^{13} \mathrm{C}$ resonance of dimethyl sulphoxide, used as solvent), and the ${ }^{1} \mathrm{H}$ methylene singlet appears at $\delta 4.87,{ }^{2}$ in strict analogy, with the ${ }^{13} \mathrm{C}$ and ${ }^{1} \mathrm{H}$ spectra of aniline and dimethylaniline.

$N$-Methylenation deeply affects the u.v. spectrum of freshly distilled (3). $\|$ Steam distillation at atmospheric pressure of (1) caused its complete hydrolysis to (3). Whereas the reduction of (1) in aqueous acidic medium with $\mathrm{NaBH}_{4}$ led to partial hydrolysis to (3) and both $N$-methyl-2,6-di-isopropylaniline and $N, N$-dimethyl-2,6-di-isopropylaniline, a result expected on the basis of the known $N$-permethylation reaction, ${ }^{5} \mathrm{LiAlH}_{4}$ rapidly converted (1) into the monomethylated compound quantitatively in tetrahydrofuran.

Addition of acetic anhydride across the azomethine bond of (1) was effected by the action of acetic acid and acetic anhydride at $130^{\circ} \mathrm{C}$ (oil bath) during $90 \mathrm{~min}$, followed by neutralization with aqueous alkali and diethyl ether extraction, to produce $N$-acetyl- $N$-acetoxymethyl-2,6-di-isopropylaniline and $N, N$-diacetyl-2,6-di-isopropylaniline in a $3: 2$ ratio. Imine (1) was converted into $N$-trifluoroacetyl- $N$ trifluoroacetoxymethyl-2,6-di-isopropylaniline by treatment with an equivalent of trifluoroacetic anhydride at room temperature during $60 \mathrm{~min}$. Reaction of (1) with either n-butylamine or n-octylamine at $75^{\circ} \mathrm{C}$ for $60 \mathrm{~min}$ caused complete reversion to the free amine (3).

Compound which showed maxima in hexane at $208(\log \varepsilon 4.32), 235$ (3.86), and $284 \mathrm{~nm}(3.43)$, with definite maxima at $206(\log \varepsilon 4.34)$ and $278 \mathrm{~nm}(2.95)$ and shoulders at $223(\log \varepsilon 3.91), 232(3.77), 298(2.88)$, and $309 \mathrm{~nm}(2.62)$.
A preliminary investigation of the system generated under analogous conditions by the reaction of (2) with 2,6dimethylaniline allowed the observation of the equilibrium between the free $N$-methylene derivative and an oligomeric species with equivalent methylene groups at room temperature by ${ }^{13} \mathrm{C}$ n.m.r. spectroscopy.

This work was supported in part by C.N.R. (grant to A. G. G.). S. C. was the recipient of a postdoctoral scholarship from Chimica del Friuli, SpA, I-33050 Torviscosa (UD).

Received, 5th August 1985; Com. 1162

\section{References}

1 G. Distefano, A. Modelli, A. G. Giumanini, and G. Poggi, J. Chem. Soc., Perkin Trans. 2, 1985, 1623

2 A. G. Giumanini, G. Verardo, L. Randaccio, and N. BrescianiPahor, J. Prakt. Chem., 1985, 327, 739

3 A. G. Giumanini, M. H. Gei, E. Zangrando, and L. Lassiani, submitted to J. Prakt. Chem.

4 M. M. Sprung, Chem. Rev., 1940, 26, 297; E. C. Wagner, J. Org. Chem., 1954, 19, 1862; R. W. Layer, Chem. Rev., 1963, 63, 489; W. V. Farrar, Rec. Chem. Progr., 1968, 29, 85; A. G. Giumanini and $\mathrm{M}$. $\mathrm{H}$. Gei, 'Aromatic and Heteroaromatic $\mathrm{N}$-Methyleneamines,' review in preparation.

5 A. G. Giumanini, M. M. Musiani, G. Chiavari, and P. Rossi, Synthesis, 1980, 9, 743.

6 B. L. Shapiro, S. J. Ebersole, G. J. Karabatsos, F. M. Vane, and S. L. Mannat, J. Am. Chem. Soc., 1963, 85, 4041 for two monomeric tertiary alkyl methyleneamines and C. F. Chang, B. J. Fairless, and M. R. Willcott, J. Mol. Struct., 1967, 22, 112, who also reported the position of the chemical shifts $(\delta 6.90-7.55)$, for methyl methyleneamine. 